\title{
Study on the Cross Compatibility Between Northwest Group and Other Cultivar Groups of Paeonia suffruticosa
}

\author{
Wang Erqiang, Ji Hanle, Liu Hongfan, Han Kun, Wang Xiaohui, Wang Zhanying* \\ Louyang Academy of Agriculture and Forestry Sciences, Louyang, China \\ Email address: \\ 1078070473@qq.com (Wang Erqiang),jihanle001@hotmail.com (Ji Hanle), liuhongfan@126.com (Liu Hongfan), \\ hankun@126.com (Han Kun), wangxiaohui@126.com (Wang Xiaohui),327109965@qq.com (Wang Zhanying) \\ ${ }^{*}$ Corresponding author
}

\section{To cite this article:}

Wang Erqiang, Ji Hanle, Liu Hongfan, Han Kun, Wang Xiaohui, Wang Zhanying. Study on the Cross Compatibility Between Northwest Group and Other Cultivar Groups of Paeonia suffruticosa. Agriculture, Forestry and Fisheries. Vol. 7, No. 5, 2018, pp. $101-107$.

doi: 10.11648/j.aff.20180705.11

Received: November 14, 2018; Accepted: December 3, 2018; Published: December 24, 2018

\begin{abstract}
Through cross experiment between Northwest group and other cultivar groups of Paeonia suffruticosa, and taking the single flower seeds as the index of affinity, the crossbreeding among Paeonia suffruticosa populations was studied. The results showed that when taking Northwest cultivar group as female parent, and crossbreed with Zhongyuan cultivar group and Japanese cultivar group respectively, no incompatibility was found, and higher average seed setting rate, 14.6 grain / flower and 9.8 grain / flower was obtained respectively. When Northwest cultivar group crossbreed with Europe and America group, there was a certain fecundity, but the seed setting rate was very low, which was 0.4 flower/seed. And there was incompatibility between Northwest cultivar group and Ito hybrid group. This study provided reference bases for the crossbreeding between Northwest group and other cultivar groups.
\end{abstract}

Keywords: Northwest Cultivar Group, Population, Hybridization, Affinity

\section{Introduction}

Peony (Paeonia suffticaosa) belongs to the Paeoniaceae, tree peonies (Paeonia Sect. Moutan) [1]. Understanding the affinity between peony varieties and cultivating new varieties of peony has become an important topic for accelerating the process of peony industrialization [2]. However, due to the relatively concentrated distribution of various varieties, the exchange of groups is limited, and the long-term hybridization work is mostly limited to repeated hybridization within a single variety group, hybrid offspring lack the participation of fresh genetics, and breeding in cross breeding within the groups is very limited.

The Northwest group has rich and varied pigmentation, cold resistance, drought resistance, strong adaptability, and strong growth characteristics, moreover, the Northwest group can be used for cutflower cultivation [3]. After the 1980s, the peony of the Northwest cultivar group was gradually discovered and established as an independent species and variety group. Its outstanding ornamental value also attracted great attention. The new varieties were selected using the seeds of natural hybrids of traditional varieties, and the germplasm of the Zhongyuan peony was also introduced into the peony of the Northwest peony variety. A large number of artificial undirected hybridization between the peony of the Northwest cultivar and the peony of the Zhongyuan group was carried out, it further enrich the inheritance of peony in the Northwest cultivar group [4-9]. A large number of new varieties were selected, but the exchange of peony in the Northwest variety group with other varieties of peony germplasm is very limited. Most of the peony species in the Northwest cultivar group have strong fertility, and have excellent characteristics such as outstanding resistance, fragrant flowers and erect heads. In the future, the peony of the Northwest cultivar group and other varieties of peony or subgenus of the same genus will be developed. The prospect of distant hybridization is very broad. It is of great significance to carry out the research on the cross-breeding of peony and inter-population peony in the Northwest cultivar group. 


\section{Materials and Methods}

\subsection{The Climate of Test Site}

Luoyang is located in the west of Henan province, it distributes in the north latitude $33^{\circ} 35^{\prime} \sim 35^{\circ} 05^{\prime}$, east longitude $111^{\circ} 8^{\prime} \sim 112^{\circ} 59^{\prime}$. It is located in the transition zone from the southern edge of the warm temperate zone to the north subtropical zone. It is a warm temperate continental monsoon climate and subtropical monsoon climate with four distinct seasons and pleasant climate. The annual average temperature is about $15^{\circ} \mathrm{C}$, the extreme maximum temperature is $40.4^{\circ} \mathrm{C}$, and the extreme minimum temperature is $-20.2^{\circ} \mathrm{C}$. The rainfall is about $630 \mathrm{~mm}$, The rainfall of the southern mountain area can reach more than $1200 \mathrm{~mm}$. The soil of the test site contained $1.74 \%$ organic matter, $0.097 \%$ total nitrogen, 21.4 $\mathrm{mg} / \mathrm{kg}$ of available phosphorus, $172.3 \mathrm{mg} / \mathrm{kg}$ of available potassium, medium fertility and medium soil.

\subsection{Test Materials}

The hybrid parent materials include the Zhongyuan peony variety group, the Northwest peony variety group, the Japanese peony variety group, the European and American peony varieties group and Ito hybrid [10]. The main features of the test materials are shown in Table 1. The hybridization work was mainly carried out in the peony resources nursery of Luoyang agriculture and forestry institute, and some pollen was provided by Luoyang national peony garden and Luoyang international peony garden.

Table 1. Characteristics of the main varieties of group.

\begin{tabular}{|c|c|c|}
\hline $\begin{array}{l}\text { Variety group } \\
\text { type }\end{array}$ & Main traits and original species & Parent material representative \\
\hline $\begin{array}{l}\text { Zhongyuan } \\
\text { variety group }\end{array}$ & $\begin{array}{l}\text { The variety is the most abundant, the plant type is high, and the degree of branch } \\
\text { development is diverse. The leaf shape changes vary and the flower color and flower } \\
\text { type are the most complete. The group is derived from long-term repeated hybridization } \\
\text { of dwarf peony, Paeonia rockii and Paeonia ostii. }\end{array}$ & $\begin{array}{l}\text { 'moshiguanyu', 'shibahao', } \\
\text { 'jinguipiaoxiang', 'heihaisajin', 'yanlongzi', } \\
\text { 'morongjiancai', 'jingyu', 'shenggejin' and so } \\
\text { on }\end{array}$ \\
\hline $\begin{array}{l}\text { Northwest } \\
\text { variety group }\end{array}$ & $\begin{array}{l}\text { The number of varieties is large, the petals base of the flower has obvious purple spots, } \\
\text { the plants are tall, the petals are thick, the flower shape evolution is lower than that of } \\
\text { Zhongyuan group, cold tolerance, drought tolerance, strong resistance. The main wild } \\
\text { species is the Paeonia suffruticosa, it has the blood relationship with the dwarf peony. }\end{array}$ & $\begin{array}{l}\text { 'yeguangbei', 'yunwushanzhuang', } \\
\text { 'zaoyuanhong', 'lanqilin', 'zhoumeigui', } \\
\text { 'lanqiangwei', 'languanyudai', } \\
\text { 'zhongchuanhuang' and so on }\end{array}$ \\
\hline $\begin{array}{l}\text { Japanese peony } \\
\text { variety group }\end{array}$ & $\begin{array}{l}\text { The flower stems are erect, the flowers are open on the leaves, and the flower structure } \\
\text { is relatively simple, the flowers are large, the colors are pure and beautiful, the } \\
\text { flowering period is late, the growth is strong, and the rate of formation is high. It was } \\
\text { introduced from China and it has been selected over a long period of time in Japan. }\end{array}$ & $\begin{array}{l}\text { 'fangji', 'taiyang', 'huawang', 'huangjing', } \\
\text { 'xugang', 'huangjiamen' and so on }\end{array}$ \\
\hline $\begin{array}{l}\text { European and } \\
\text { American peony } \\
\text { variety group }\end{array}$ & $\begin{array}{l}\text { Most of the varieties have large flowers, strong petal, and the flowers are sideways or } \\
\text { drooping. They grow vigorously and have many secondary flowering habits. The } \\
\text { flowering period is particularly late and the adaptability is strong. It belongs to distant } \\
\text { hybrid and it is the hybridization of Zhongyuan peony from China, Japanese peony and } \\
\text { wild peony. }\end{array}$ & $\begin{array}{l}\text { 'haihuang', 'jinge', 'ruinong', ‘jinhuang', } \\
\text { 'jinyang', 'heibao' and so on }\end{array}$ \\
\hline Ito hybrid & $\begin{array}{l}\text { The flowering period is very lateand long. It belongs to the distant hybrid between } \\
\text { Paeonia suffruticosa and Paeonia lactiflora. }\end{array}$ & 'dongfangjin' and 'huayuanzhenbao' \\
\hline
\end{tabular}

\subsection{Configuration of Hybrid Combinations Between Varieties}

Northwest variety group $\times$ Zhongyuan variety group; Northwest variety group $\times$ Japanese variety group, Northwest variety group $\times$ European and American variety group; Northwest variety group $\times$ Ito hybrid.

\subsection{Test Methods}

Conventional cross breeding methods are employed in the test. The selected female parent is fertile. Pollination begins when the stigma begins to secrete mucus. Pollination was carried out before $10 \mathrm{am}$ or after $17 \mathrm{am}$, and pollinating 3 times everyday for 3 days continuously. The spare pollen is labeled and placed in a refrigerator at $-20^{\circ} \mathrm{C}$ for storage. And the hybrid combination was tagged (11xxx is the 2011 configuration combination, $12 \mathrm{xxx}$ is the 2012 configuration combination, and $13 \mathrm{xxx}$ is the 2013 combination).

The pod was removed when the color of the female pod is crab yellow and micro-cracked and placed it in a cool and ventilated place, waited for the pod to naturally crack, afterwards peeled off the seeds and counted the number of seeds, and calculated the setting percentage. The average number of seed of individual flower in a single combination reflects the hybridization affinity of a single combination, and the average number of seed of individual flower in the populations reflects the strength of overall hybridization affinity.

\section{Results and Analysis}

\subsection{Analysis of Hybrids Affinity Between Northwest Variety Group and Zhongyuan Variety Group}

In 2011-2013, 31 varieties peony of the Northwest cultivar group were used as the female parent, and 24 varieties peony of the Zhongyuan group were used as the male parent. A total of 39 hybrid combinations were arranged. It can be seen in Table 2. The average setting percentage of per flower of each combination is significantly different, and the average setting percentage of per flower of each combination is 0.2-42.1 per grain or flower, and the largest setting percentage of the combination is 12074 ('xiongmao' $\times$ 'shibahao'), which the average setting percentage is 42.1 grain/flower, and the combination that the setting percentage of per flower less than 
1 is 13069 ('fenzouchou'×'moyujuelun'), 13036 ('moguanyuzhu'x'yanrongzi') and 13031 ('Xian e' mao $\times$ 'molouzhenghui'), the setting percentage of per flower is 1.0 grain/flower, 0.9 grain/flower and 0.2 grain/flower respectively. At the same time, we found that the setting percentage of the combinations was different, including the combinations with different paternal parent, the same female parent or different female parent, the same male parent. It can be seen that there is no cross-breeding incompatibility between the peony of the Northwest cultivar and the peony of the Zhongyuan cultivar, the cross-breeding between the two peony group belongs to the close hybridization. However, the setting percentage varies greatly among the combinations, and the hybrid affinity between the varieties is not consistent. The male and female parent influenced the level of affinity together.

Table 2. The results of hybridization with Northwest cultivar group as female parent and Zhongyuan cultivar group as male parent of peony.

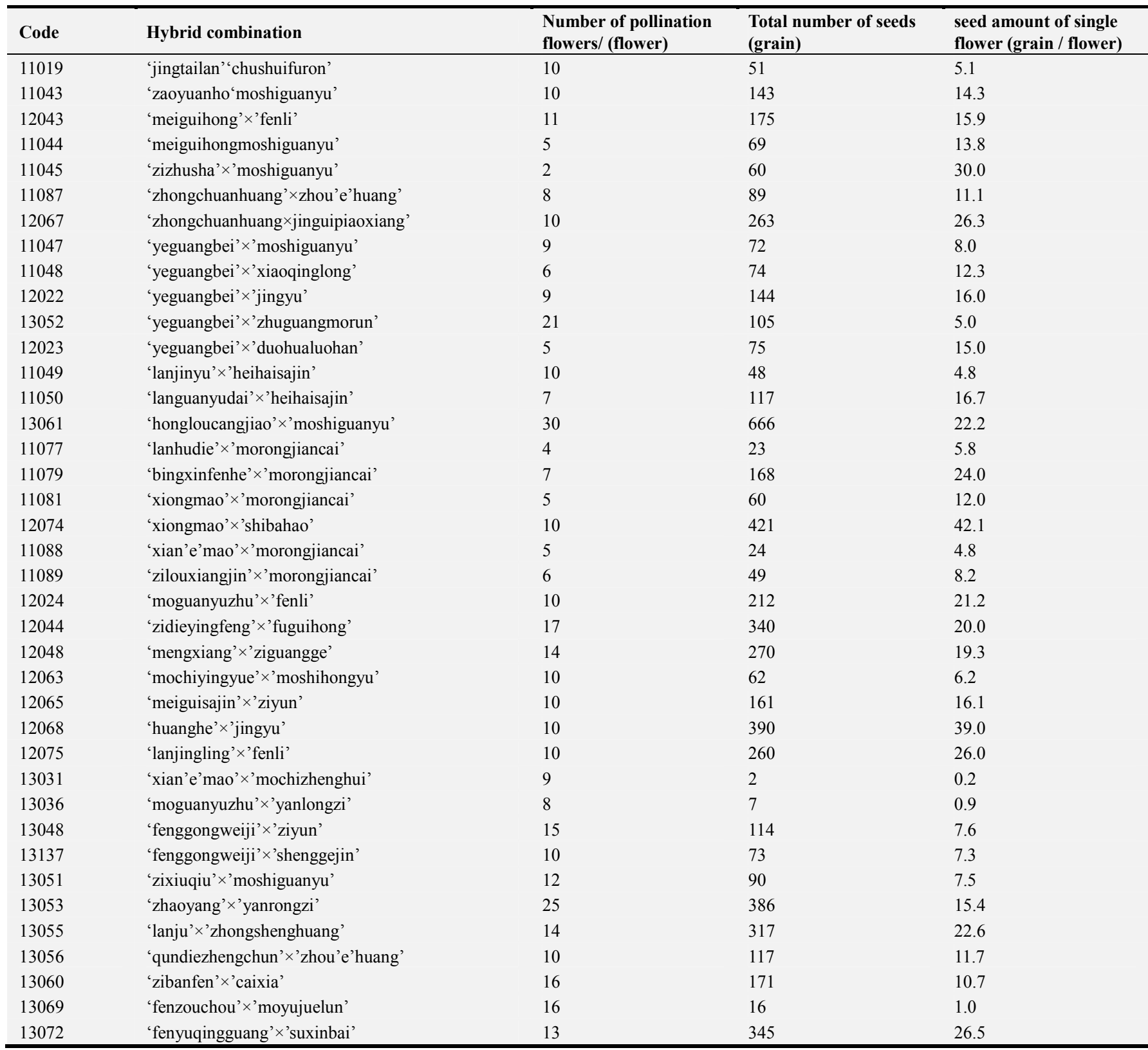

\subsection{Affinity Analysis of Crossbreeding of Northwest Varieties Peony and Japanese Varieties of Peony}

In 2011 and 2013, a total of 36 combinations were crossed, including 25 varieties peony of the Northwest variety, and 13 varieties of Japanese peony variety group. As shown in Table 3. The differences among the combinations were large, and the setting percentage of combination was 0.7-37 grain/flower. The combination with the highest seed setting percentage was 13073 ('fenyuqingguang' $\times$ 'daodachen'), the setting percentage was 37.0 grain / flower, followed by 12093 ('lanqiangwei ' $\times$ 'taiyang') 26.5 grain / flower, the lowest seed setting percentage was for 12087 ('longyuanhong' $\times$ 'xugang') 0.7 grain / flower, followed by 13035 ('yeguangbei' $\times$ ' fangji') 1.5 grain / flower. There is a significant difference in the 
setting percentage of the combination of the same female parent but different male parent. For example, the seed setting percentage of the five combinations that 'zaoyuanhong' as the female parent, 'xugang'and 'fangii' as the male parent with a high setting percentage of 12.7 grain / flower, 11.3 grain / flower respectively, and when 'qunwu' as the male parent, the setting percentage is low (2.2 grain / flower). Similarly, the difference in the seed setting rate of the combination of the same male parent but different female parent is also very obvious. For example, the seed setting percentage of the eight combinations that 'fangji' as the male parent, the combination with the highest setting percentage (12.2 grain / flower) was 11091 ('zidieyingfeng' $\times$ 'fang ii'), the combination with the lowest seed setting percentage (only 1.5 grain / flower) is 13035 ('yeguangbei'×'fangji'), only 1.5 grain / flower. However, there is no unconformity in all combinations. It can be seen that there is no hybrid incompatibility between the peony of the Northwest cultivar and the Japanese cultivar group, it belongs to the close hybridization. The paternal pollen activity of the male parent and fertility of female parent affect the setting percentage of each combination together. In addition, the setting percentage of the same combination was significantly different in different years, it may be related to the climate and the development of the flower itself in the current year. It is necessary to further study with multi factors.

Table 3. The results of hybridization with Northwest cultivar group as female parent and Japanese peony variety group as male parent of peony as male parent.

\begin{tabular}{|c|c|c|c|c|}
\hline Code & Hybrid combination & $\begin{array}{l}\text { Number of pollination } \\
\text { flowers (flower) }\end{array}$ & $\begin{array}{l}\text { Total number of seeds } \\
\text { (grain) }\end{array}$ & $\begin{array}{l}\text { Seed amount of single } \\
\text { flower (grain / flower) }\end{array}$ \\
\hline 11041 & 'mochiyingyue'×'xinriyuejin' & 4 & 73 & 18.3 \\
\hline 12062 & 'mochiyingyue'×'xinriyuejin' & 10 & 83 & 8.3 \\
\hline 11084 & 'moguanyuzhu' $\times$ 'taiyang' & 5 & 26 & 5.2 \\
\hline 11091 & 'zidieyingfeng'×'fangji' & 5 & 61 & 12.2 \\
\hline 12041 & 'zaoyuanhong'×'fangji' & 9 & 102 & 11.3 \\
\hline 12042 & 'zaoyuanhong' $\times$ 'tongyun' & 18 & 105 & 5.8 \\
\hline 13135 & 'zaoyuanhong'×'qunwu' & 13 & 28 & 2.2 \\
\hline 13136 & 'zaoyuanhong'×'taiyang' & 15 & 64 & 4.3 \\
\hline 11090 & 'zaoyuanhong'×'xugang' & 10 & 127 & 12.7 \\
\hline 12073 & 'xuezhongsongtan'×'baifeng' & 13 & 190 & 14.6 \\
\hline 12087 & 'longyuanhong'×'xugang' & 10 & 7 & 0.7 \\
\hline 12088 & 'zhoumeigui' $\times$ 'xugang' & 10 & 42 & 4.2 \\
\hline 12090 & 'ziyunxian'×'huawang' & 13 & 146 & 11.2 \\
\hline 13046 & 'zilouxiangjin'×'huawang' & 10 & 104 & 10.4 \\
\hline 12092 & 'zilouxiangjin'×'huawang' & 11 & 210 & 19.1 \\
\hline 13068 & 'lanqiangwei'×'fangji' & 20 & 260 & 13.0 \\
\hline 12093 & 'lanqiangwei' $\times$ 'taiyang' & 10 & 265 & 26.5 \\
\hline 12145 & 'mingmou' $\times$ 'mingshixie' & 10 & 47 & 4.7 \\
\hline 12146 & 'mingmou' $\times$ 'daodachen' & 10 & 34 & 3.4 \\
\hline 13032 & 'yunwushanzhuang'×'fangji' & 10 & 99 & 9.9 \\
\hline 13054 & 'mengxiang'×'fangii' & 18 & 210 & 11.7 \\
\hline 13033 & 'mengxiang'×'fangji' & 16 & 157 & 9.8 \\
\hline 13035 & 'yeguangbei'×'fangji' & 20 & 30 & 1.5 \\
\hline 13037 & 'zizhusha'×'fangji' & 11 & 70 & 6.4 \\
\hline 13038 & 'hepinghong' $\times$ 'xinriyuejin' & 9 & 96 & 10.7 \\
\hline 13049 & 'saihuanghou' $\times$ 'daojin' & 12 & 140 & 11.7 \\
\hline 13050 & 'ziyan'×'daojin' & 11 & 178 & 16.2 \\
\hline 13057 & 'qundiezhengchun'×'taiyang' & 16 & 211 & 13.2 \\
\hline 13058 & 'taohuafen'x'daojin' & 15 & 70 & 4.7 \\
\hline 13059 & 'taohuafen'×'daodachen' & 11 & 38 & 3.5 \\
\hline 13062 & 'hongloucangjiao'×'fangji' & 11 & 85 & 7.7 \\
\hline 13063 & 'hongyangfei' $\times$ 'fangji' & 15 & 152 & 10.1 \\
\hline 13071 & 'fenzouchou'×'huawang' & 10 & 80 & 8.0 \\
\hline 13073 & 'fenyuqingguang' $\times$ 'daodachen' & 10 & 370 & 37.0 \\
\hline 13074 & 'qingchun'×'huajing' & 10 & 61 & 6.1 \\
\hline 13075 & 'qingchun' $\times$ 'huangjiamen' & 10 & 97 & 9.7 \\
\hline
\end{tabular}

\subsection{Analysis of the Hybridity of Peony in the Northwest Cultivar Group and the European and American Varieties}

There were 6 varieties of European and American varieties peony which belonged tothe hybrids of peony subgroups, and 9 varieties peony of the Northwest variety group in the experiment. 10 combinations were arranged. As shown in Table 4, the combination 'huanghe' $\times$ 'haihuang', 'zaoyuanhong' $\times$ 'mingwang', 'Yuxiu' $\times$ 'mingwang', 
'fenzouchou' $\times$ 'ruinong', 'meiguihong ' $\times$ ' Jinyang' has a certain amount of seed, but the seed setting rate is low, and the 'fenzouchou' $\times$ 'ruinong' has a highest setting rate of 1.5 grain/flower, indicating that this combination is higher affinity. Other combinations have no seed. The results showed that this distant hybrid type can obtain seeds, but the seeding rate is significantly different due to different hybrid combinations. It can be seen that hybrids between the peony of the Northwest cultivar group and hybrids of infra-subsectional can obtain seeds and have certain hybrid affinity, but the rate of seed setting is significantly lower than that of the infra-sectional close hybridization.

Table 4. The results of hybridization withNorthwest cultivar group as female parent and Europe and America cultivar group as male parent.

\begin{tabular}{|c|c|c|c|c|}
\hline Code & Hybrid combination & $\begin{array}{l}\text { Number of pollination } \\
\text { flowers (flower) }\end{array}$ & $\begin{array}{l}\text { Total number of seeds } \\
\text { (grain) }\end{array}$ & $\begin{array}{l}\text { Seed amount of single } \\
\text { flower (grain / flower) }\end{array}$ \\
\hline 11110 & 'huanghe' ×'haihuang' & 30 & 5 & 0.2 \\
\hline 11111 & 'zhongchuanhuang'×‘jinge' & 30 & 0 & 0.0 \\
\hline 11137 & 'zaoyuanhong'×‘mingwang' & 25 & 16 & 0.6 \\
\hline 12078 & 'yeguangbei’×‘heibao' & 25 & 0 & 0.0 \\
\hline 12082 & 'mingxiu'×'mingwang' & 30 & 28 & 0.9 \\
\hline 12083 & 'xueranfengcai' $\times$ 'haihuang' & 30 & 15 & 0.5 \\
\hline 13070 & 'fenzouchou'×'ruinong' & 16 & 24 & 1.5 \\
\hline 13087 & 'hepinghong'×'haihuang' & 15 & 0 & 0.0 \\
\hline 13098 & 'meiguihong'×'jinyang' & 20 & 8 & 0.4 \\
\hline 13106 & zhongchuanhuang'×'jinyang' & 30 & 0 & 0.0 \\
\hline
\end{tabular}

\subsection{Analysis of the Hybridity of Peony in the Northwest Cultivar Group and the Ito Hybrids Varieties}

The selected type of Ito hybrids in this experiment was 'dongfangjin'and 'huayuanzhenbao'. A total of 9 hybrid combinations were arranged. 205 flowers were pollinated. None of the combinations received normal seeds. The results showed that there was no hybrid affinity between Ito hybrids and Northwest cultivar group. Incompatibility may be related to the pollen vigor of male parent and hybrid combination used. Compared with the previous literature that "Ito hybrids as inter-species hybrids of peony and Chinese herbaceous peony, their male parent have certain fertility, although stamens have less powder, but some varieties with more powder and Chinese herbaceous peony (or peony) crossed and can obtain seed" is inconsistent and need further research.

Table 5. The results of hybridization with Northwest cultivar group as female parent and Ito hybrid varieties as male parent.

\begin{tabular}{|c|c|c|c|c|}
\hline Code & Hybrid combination & $\begin{array}{l}\text { Number of pollination } \\
\text { flowers (flower) }\end{array}$ & $\begin{array}{l}\text { Total number of seeds } \\
\text { (grain) }\end{array}$ & $\begin{array}{l}\text { Seed amount of single } \\
\text { flower (grain / flower) }\end{array}$ \\
\hline 12183 & 'ganlanyu' $\times$ 'dongfangjin' & 20 & 0 & 0 \\
\hline 12184 & 'bingshanfeicui'×'dongfangjin' & 20 & 0 & 0 \\
\hline 12185 & 'lanhe'×’huayuanzhenbao' & 20 & 0 & 0 \\
\hline 12186 & 'dabanlan'×'huayuanzhenbao' & 35 & 0 & 0 \\
\hline 13141 & 'yinhongfeihe'×'dongfangjin' & 20 & 0 & 0 \\
\hline 13142 & 'zibanfen'×'huayuanzhenbao' & 20 & 0 & 0 \\
\hline 13143 & 'sanzhuan×'huayuanzhenbao' & 20 & 0 & 0 \\
\hline 13145 & 'lanhudie'×'huayuanzhenbao' & 30 & 0 & 0 \\
\hline 13146 & 'bingxinfenhe' $\times$ 'dongfangjin' & 20 & 0 & 0 \\
\hline
\end{tabular}

\subsection{Analysis of the Average Seed Setting Rate of Hybrids Between Northwest Cultivar Group and Other Cultivar Group}

The Northwest cultivar as the female parent and species of other peony cultivar groups as the male parent, the average seed setting rate of each combination were obtained and the results showed in Fig. 1. It can be seen from the figure that the order of average seed setting rate of the combinations of the Northwest cultivar group as the female parent, and the other varieties as male parent: Zhongyuan peony variety group $>$ the
Japanese variety group $>$ the European and American variety group $>$ Ito hybrid. Among the above arranged combinations, the highest seed setting rate of the peony of Zhongyuan group was 14.6 grain/flower, and the setting rate of the Japanese peony group was 9.8 grain/flower. The results showed that there was no incompatibility existed in the hybrid of species of the Northwest cultivar group with Zhongyuan group and the Japanese peony group. It belongs to close hybridization. However, the hybridization between the European and American varieties of peony and Ito hybrids is poor or not strong, and it belongs to distant hybridization. 


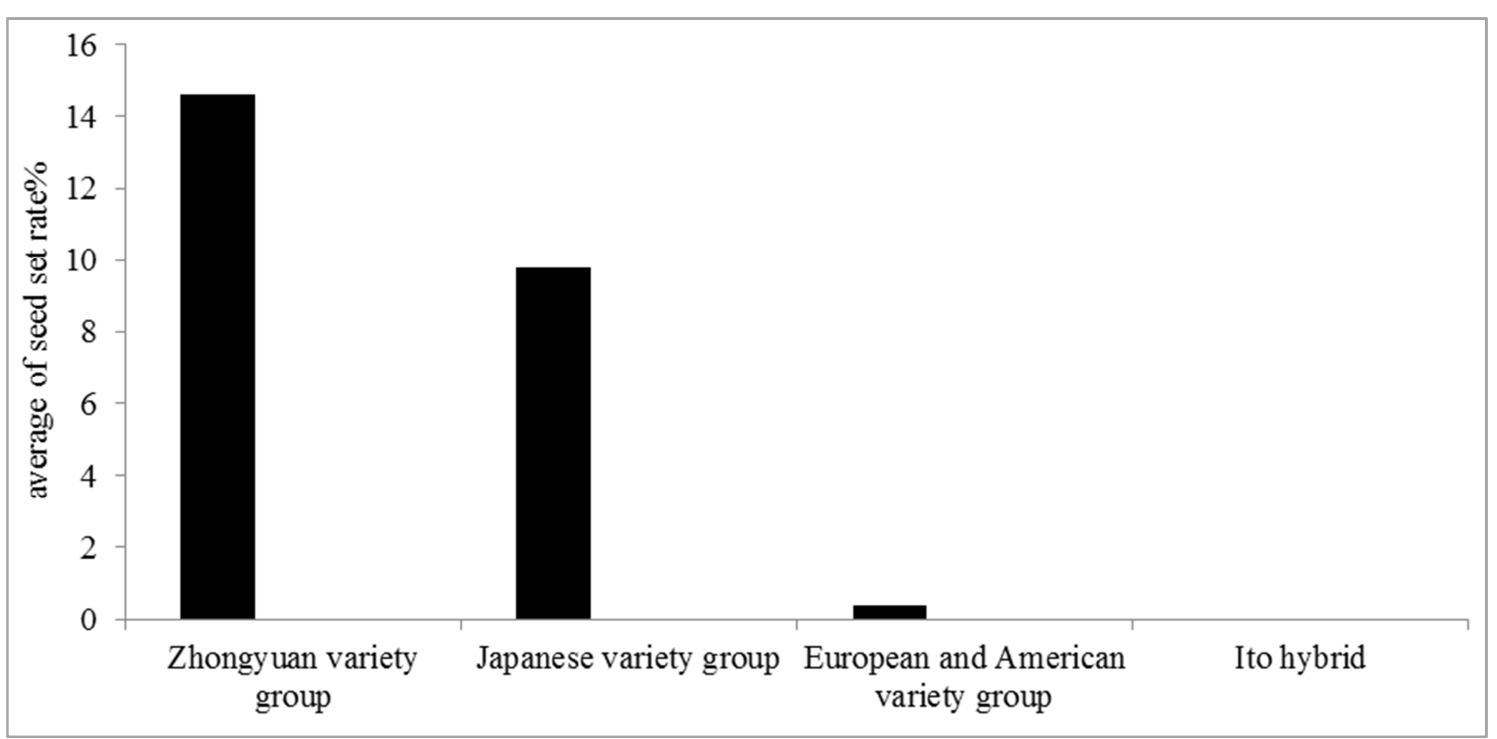

Figure 1. The average seed setting rate of Northwest cultivar group of tree peony as female parent when different cultivar group as the male parent.

\section{Discussion}

In this study, some seeds stopped growing or normal seeds could not germinate during development. Combining peony embryo culture [11-15] to realize plant regeneration technology will be an effective means to solve the problem that peony distant hybrids are not developed and seeds are not germinated. Meanwhile, when the peony of the Northwest cultivar as the female parent and Ito hybrids as the male parent, no hybrids were obtained, which showed that the peony of the Northwest cultivar and Ito hybrids had reproductive isolation, and the genetic relationship between the cultivars was far. It may be due to parent materials selected, combinations arranged, and the number of hybrid populations.

\section{Conclusion}

When the peony of the Northwest cultivar group as the female parent, the peony of the Zhongyuan group and the peony of the Japanese cultivar group as the male parents, all of the combinations had higher seed setting rate, and there was no hybrid incompatibility between the combinations, which showed that genetic relationship among these varieties group is relatively close, corresponding to the large overlap of the original species composition of these varieties. However, the difference in seed setting rate of different varieties is obvious. It can be seen that the maternal fertility and the affinity of the parents' hybrids affect the seed setting rate together.

When the peony of the Northwest cultivar group as the female parent, the peony of the European and American variety group as the male parents, all of the combinations had low seed setting rate or no seed, and there was hybrid incompatibility existed. This showed that these varieties have far genetic relationship, and the hybridization is distant hybridization. However, through the large number of crosses made between the two varieties group, some combinations with relatively high seeding rate can be obtained, and the seedlings of the offspring are used to breed new, exotic and special peony varieties.

When the peony of the Northwest cultivar as the female parent and Ito hybrids as the male parent, there is no strong combinations screened in this study, In order to conduct a more in-depth study on the hybrid affinity of peony of Northwest cultivar group and Ito hybrids, it is necessary to increase the number of parental materials, optimize the combination schemes, and expand the number of hybrid populations in further study.

\section{References}

[1] J. J. Li, L. X. He, F. H. Chen, et al. Preliminary report on flowering pollination biology and artificial hybridization of Peony peony [J]. Plant Introduction and Domestication, 1997 (11): $65-72$.

[2] D. L. Guo. Advances in Genetic Diversity of Peony Germplasm Resources [J]. Northern Horticulture, 2007 (9): 61-65.

[3] J. J. Li. Chinese Peony [M]. Beijing: China Encyclopedia Publishing House, 2011.

[4] F. Y. Cheng, J. J. Li, D. Z. Chen, et al. Chinese Paeonia suffruticosa [M]. China Forestry Publishing House, 2005.

[5] N. Zhao. Study on the morphology and karyotype of progeny and parents of peony distant hybrids [D]. Beijing Forestry University, 2014.

[6] X. Han Selection of Peony Hybrid Parents and Genetic Expression of F1 Generation [D]. Beijing Forestry University, 2014.

[7] Yang Kunmei. Study on the genetic relationship of 96 different species of peony varieties [D]. 2015.

[8] X. M. Sun, J. Q. Cui, H. C. Wang, et al. Hybrid affinity of Paeonia and molecular identification of hybrid progeny ISSR [J]. Northern Horticulture, 2016 (9): 98-101. 
[9] E. Q. Wang, Z. Y. Wang, J. J. Pang, et al. Analysis on cross compatibility and breeding utilization value of 'yeguangbei' cultivar group of Paeonia rockii [J]. Journal of Henan University of Science and Technology (Natural Science), 2017, 45 (1): 14-19.

[10] G. X. Wu, L. H. Cui, S. H. Liu, et al. Demonstration research on introduction and cultivation of foreign Ito hybrid peony [J]. Northern Horticulture, 2001 (24): 67-71.

[11] L. Y. Wang. Chinese peony variety map [M]. Beijing: China Forestry Publishing House, 1997.

[12] A. L. An, X. L. Su, J. Mao, et al. A study on immature embryo culture of Paeonia rockii in vitro [J]. Journal of Gansu Agricultural University, 2009 (6): 63-68.

[13] R. C. Zhou, C. H. Yao, Embryo culture and plantlet regeneration of Paeoniarockii [J]. Subtropical Plant Science, 2001, 30 (3): 60.

[14] H. C. Yang, D. L. Pei, Study on Embryo Culture of Peony Seeds [J]. Guangxi Agricultural Sciences, 2006, 37 (2): $108-110$.

[15] L. Xu, F. Y. Cheng, Y. Z. Study on Rapid Seedling-raising Technology of Tree Peony Embryo Culture [J]. Bulletin of Botanical Research, 2017. 